\title{
Measuring the Performance of Image Contrast Enhancement Technique
}

\author{
Dominic Asamoah \\ Department of Computer \\ Science, KNUST, Ghana
}

\author{
Emmanuel Ofori \\ Oppong \\ Department of Computer \\ Science, KNUST, Ghana
}

\author{
Stephen Opoku \\ Oppong \\ Faculty of Computing and \\ Information Systems, \\ GTUC, Ghana
}

\author{
Juliana Danso \\ Department of Computer \\ Science, KNUST, Ghana
}

\begin{abstract}
Image enhancement is one of the key techniques in processing quality of images in systems. The main purpose of image enhancement is to bring out detail that is hidden in an image or to increase contrast in a low contrast image. This technique provides a multitude of choices for improving the visual quality of images. This is the main reason that image enhancement is used in a huge number of applications with important challenges such as noise reduction, degradations, blurring etc. This paper focuses on three contrast enhancement techniques for image enhancement which are: Histogram Equalization (HE), Adaptive Histogram Equalization (AHE) and Contrast Limited Adaptive Histogram Equalization (CLAHE) which are then compared with the help of the eight (8) quality image measurement metrics which are: i.e. the Mean squared error (MSE), Root Mean squared error (RMSE), Peak signal noise ratio (PSNR), Mean absolute error (MAE), Signal to noise ratio (SNR), Image Quality Index (IQI), Similarity Index (SI) and Pearson Correlation Coefficient ( $r$ ). The paper concluded that Histogram Equalization (HE), is the one best contrast enhancement technique, as it recorded high percentage values for all the eight (8) quality image measurement metrics. Overall, it was therefore recommended histogram equalization technique should be embedded in any system that processes on images and output them to humans, for making lifechanging decisions
\end{abstract}

\section{General Terms}

Computer Graphics, Image Processing

\section{Keywords}

Image Enhancement, Contrast, Histogram equalization, Measurement Metrics, Noise Reduction

\section{INTRODUCTION}

The goal of image enhancement is to improve the image quality so that the processed image is better than the original image for a specific application or set of objectives. Many image enhancement algorithms have been proposed. One of the most widely used algorithms is contrast histogram equalization, which adjusts the intensity histogram to approximate a uniform distribution. The main disadvantage of histogram equalization is that the image properties may not be appropriately applied in a local context. Another disadvantage of this method is that it is indiscriminate. Thus, it may increase the contrast of background noise, while decreasing the usable signal. In fact, histogram modification treats all regions of the image equally and, thus, often yields poor local performance in terms of detail preservation. Therefore, several local contrast histogram equalization algorithms have been introduced to improve image enhancement.
The study will therefore take a look into other two advanced histogram equalization methods, which are the adaptive histogram equalization (AHE) and the contrast limited adaptive histogram equalization (CLAHE). The algorithms described are contrast enhancement methods that enhance the images by improving the contrast measurement directly. Again, the study will measure the extent to which these contrast enhancement methods visually and digitally optimizes the optical appearance of the processed image. The problem of enhancing contrast of images enjoys much attention and spans a wide gamut of applications, ranging from improving visual quality of photographs acquired with poor illumination [1], [2], to medical imaging [3]. Common techniques for global contrast enhancements, like global stretching and histogram equalization, do not always produce good results, especially for images with large spatial variation in contrast.

\section{LITERATURE REVIEW}

Image Enhancement is simple and most appealing area among all the digital image processing techniques. The main purpose of image enhancement is to bring out detail that is hidden in an image or to increase contrast in a low contrast image. Whenever an image is converted from one form to other such as digitizing the image some form of degradation occurs at output. Contrast is a necessary factor when it comes to processing images.

Contrast is the difference in visual properties that makes an object (or its representation in an image) distinguishable from other objects and the background. In visual perception of the real world, contrast is determined by the difference in the color and brightness of the object and other objects within the same field or view. In other words, it is the difference between the darker and the lighter pixel of the image, if the difference is big the image will have high contrast and if it is small the image will have a low contrast. In this paper, performances of the various image contrast techniques which are suitable for real life applications is compared. These experimented techniques are mostly histogram adjustment methods. Performances of these methods are compared with common objective quality metrics on different image set [4].

\subsection{Image Contrast Enhancement Technique}

The principal objective of enhancement is to process an image so that the result is more suitable than the original image for a specific application. The word specific is important, because it establishes at the outset that the techniques are oriented to the problem. Thus, for example, a method that is quite useful for enhancing X-ray images may not necessarily be the best approach for enhancing pictures of an unborn baby transmitted by an ultra-scan machine. Regardless of the method used, however, image enhancement is one of the most 
interesting and visually appealing areas of image processing. When an image is processed for visual interpretation, the viewer is the ultimate judge of how well a particular method works. Visual evaluation of image quality is a highly subjective process, thus making the definition of a "good image" an elusive standard by which to compare algorithm performance. When the problem is one of processing images for machine perception, the evaluation task is somewhat easier. For example, in dealing with a character recognition application, and leaving aside other issues such as computational requirements, the best image processing method would be the one yielding the best machine recognition results. However, even in situations when a clearcut criterion of performance can be imposed on the problem, a certain amount of trial and error usually is required before a particular image enhancement approach is selected [5].

\subsection{Histogram Equalization}

This contrast enhancement method uses the concept of spreading pixel values in the given image to the entire histogram more uniformly. This way, the unseen scene details are more visible to the observer. Thus, HE uses the histogram of the input image and brings it to its ideal uniform form. Histogram is constructed by calculating the pixel frequencies for the entire dynamic range of the input image. Pixel frequency represents the number of times a gray level seen in the input image. The histogram equalization method has less computational complexity. However, it is also clear that this method converts the pixel values with higher-frequency counts to the very large gray level intervals. This means that small details disappear between the objects with high frequencies (background, large objects, etc.) at the output image [6].

\subsection{Adaptive Histogram Equalization/ Image Adjustment}

This is an extension to traditional Histogram Equalization technique. It enhances the contrast of images by transforming the values in the intensity image. Unlike HE, it operates on small data regions (tiles), rather than the entire image. Each tile's contrast is enhanced, so that the histogram of the output region approximately matches the specified histogram. The neighboring tiles are then combined using bilinear interpolation in order to eliminate artificially induced boundaries. AHE method divides an input image to two sub images based on the mean histogram frequency value and enhances them separately. It can enhance contrast by preventing the significant change in gray level. This method has better output performance compared to HE. However, it has a higher computational load. The contrast, especially in homogeneous areas, can be limited in order to avoid amplifying the noise which might be present in the image [7].

\subsection{Contrast-Limited Adaptive Histogram Equalization}

The CLAHE method algorithm is used for contrast enhancement. It divides the original image into several nonoverlapping sub images and the histograms for each are derived. Histograms of sub images are cut at some threshold to limit the amount of enhancement and then equalized. The details of the image appear clearly relative to the background. At the same time, background is also enhanced as the foreground thus leading to a high contrast image.

In medical images, CLAHE effectively enhances the local features and the image contrast while suppressing the noise. It also preserves the richness of details and the sharpness of edges. Compared to Histogram Equalization, it takes less computational time. CLAHE method calculates the histogram of the whole image, and then the histogram is clipped according to the threshold value. After this, it uses the excess frequency counts and distributes them uniformly over the input histogram interval. This operation adds the unused histogram bins of the input image to the mapping calculation and limits the enhancement operation. This way, the resulting image looks more natural [8].

\subsection{Related Works}

Image enhancement process consists of a collection of techniques that seek to improve the visual appearance of an image or to convert the image to a form better suited for analysis by a human or machine. The principal objective of image enhancement is to modify attributes of an image to make it more suitable for a given task and a specific observer. During this process, one or more attributes of the image are modified. Digital Image enhancement techniques provide a multitude of choices for improving the visual quality of images [9]. Appropriate choice of these techniques is influenced by the related task, the image modality and viewing condition. It is important to keep in mind that enhancement is a very subjective area of image processing. Improvement in quality of these degraded images can be achieved by using application of enhancement techniques. The work done by various researchers for Image Enhancement are discussed here, Madhu [5] suggested that the Adaptive histogram equalization produced a better result, but the image is still not free from washed out appearance. Tang suggested global histogram equalization, which adjusts the intensity histogram to approximate uniform distribution [10]. The global histogram equalization is that the global image properties may not be appropriately applied in a local context. In fact, global histogram modification treats all regions of the image equally and, thus, often yields poor local performance in terms of detail preservation. Therefore, several local image enhancement algorithms have been introduced to improve enhancement [11].

\section{METHODOLOGY}

This paper investigates into three image contrast enhancement techniques which are image adjustment, histogram equalization and contrast-limited adaptive histogram equalization, in relation to improving the quality of images. The extent to which each of these three contrast enhancement techniques enhances images, are then compared with each other using eight (8) performance metrics which are PSNR, MSE, MAE, IQI, RMSE, SI, SNR, and Statistical Pearson Correlation Error (r).[12]

\subsection{Performance Metrics}

Performance metric are used to quantitatively measure the features of an image. It can also describe, classify and, produce change detection in the image. For the purposes if this study, the performance metrics reviewed is to extract certain features of an enhanced image included Mean Square Error (MSE), Root Mean Square Error, Peak Signal to Noise Ratio (PSNR), Mean Absolute Error (MAE) Signal to Noise Ratio (SNR), Image Quality Index (IQI), Similarity Index (SI) and Statistical Pearson Correlation Coefficients (r)

\subsubsection{Mean Squared Error (MSE)}

The Mean Square Error (MSE) is the mean squared error between the filtered image and the original image. Mean Square Error can be estimated in one of many ways to quantify the difference between values implied by an estimate 
and the true quality being certificated. MSE is a risk function corresponding to the expected value of squared error. The MSE is the second moment of error and thus incorporates both the variance of the estimate and its bias. MSE is given by the formula below, where $\mathrm{M}$ and $\mathrm{N}$ is the width and the height of the images, enhanced image $I(i, j)$ and the original image $K(i, j)$. The $i$ and $j$ are the row and column pixels of both the original and enhanced images.

$$
M S E=\frac{1}{M * N} \sum_{i=0,}^{M-1, N=0}{ }_{j=0}[I(i, j)-K(i, j)]^{2}
$$

When two images are identical, the MSE will be zero. For this value the PSNR is undefined (Division by zero). An identical image to the original will yield an undefined PSNR as the MSE will become equal to zero due to a division-by-zero error. [13]

\subsubsection{Peak Signal to Noise Ratio (PSNR)}

The Peak Signal to Noise Ratio (PSNR) is the ratio between maximum possible power and corrupting noise that affect representation of image. In other words, PSNR is an engineering term for the ratio between the maximum possible power of a signal and the power of corrupting noise that affects the fidelity of its representation. PSNR is usually expressed as decibel scale. Because many signals have a very wide dynamic range, PSNR is usually expressed in terms of the logarithmic decibel scale and the performance of the proposed algorithms was evaluated in terms of the visual quality and the peak-signal-to-noise-ratio (PSNR). The PSNR is commonly used as measure of quality reconstruction of image. The signal in this case is original data and the noise is the error introduced. High value of PSNR indicates the high quality of image. It is defined via the Mean Square Error (MSE) and corresponding distortion metric. The following methodology to find out the PSNR value for a given gray image is used:

$$
\begin{aligned}
& P S N R=10 \log _{10}\left[\frac{M A X_{i}{ }^{2}}{M S E}\right] \\
& P S N R=20 \log _{10}\left[\frac{M A X_{i}}{M S E}\right]
\end{aligned}
$$

$P S N R=20 \log _{10}\left(M A X_{i}\right)-10 \log _{10}(M S E)$ Where, MAXi is the maximum possible pixel value of the image. When the pixels are represented using 8 bits per sample, MAXi is 255. More generally, when samples are represented B bits per sample, MAXi is $2 \mathrm{~B}-1$ and MSE is Mean Square Error between the filtered image and the original image. [14]

\subsubsection{Root Mean Squared Error (RMSE)}

The Root Mean Square Error (RMSE) is given by as the squared root of MSE. The root mean square error (RMSE) measures the amount of change per pixel due to the processing. The RMSE between a reference or original image, image $1-K(i, j)$ and the enhanced image, image2- $I(i, j)$ is given by

$$
\begin{aligned}
& R M S E=\sqrt{\frac{1}{M * N} \sum_{i=0, j=0}^{M-1, N-1}[I(i, j)-K(i, j)]^{2}} \\
& \text { Or simply, }
\end{aligned}
$$

$$
R M S E=\sqrt{M S E}
$$

This shows that a higher PSNR and higher value of MSE \& RMSE provides a higher image quality. PSNR, MSE and RMSE can be used to evaluate the quality of an image, the bigger the values for these metrics, the less distortion. MSE is smaller, the performance is worse, which means the filtered image is close to the original and similarity index is very high, closer to 1. [13]

\subsubsection{Signal to Noise Ratio (SNR)}

The signal-to-noise ratio, SNR, can have several definitions. Signal to Noise Ratio is defined by the power ratio between a signal and the background noise. SNR is calculated by the formula below where $\mathrm{P}$ is average power. Both noise and power must be measured at the same points in a system, and within system with same bandwidth. Generally, if the SNR is bigger and positive (i.e. $\mathrm{SNR}>0$ ), the noise is less.[13]

$$
S N R=\frac{P_{\text {signal }}}{P_{\text {noise }}}
$$

SNR (Signal to Noise Ratio) in decibels is defined as the formula below where $\sigma 2$ is the variance of the noise free image and $\sigma \mathrm{c} 2$ is the variance of enhanced image.

$$
S N R_{d B}=10 \log _{10}\left(\frac{\sigma^{2}}{\sigma_{c}^{2}}\right)
$$

Noise appearance is inherent in amplitude quantization process where analog data such as an image is being converted into digital data. In this noise model, the signal-tonoise ratio (SNR) is limited by minimum and maximum pixel value, Pmin and Pmax respectively and the noise is characterized by its standard deviation, Sn. The characterization of the signal can then differ. If the standard deviation of the noise is known to lie between two signal boundaries, $\mathrm{P} \min \leq \mathrm{Sn} \leq \mathrm{P} \max$, then the $\mathrm{SNR}$ is defined as:

$$
S N R_{d B}=20 \log _{10}\left(\frac{\left(P_{\max }-P_{\min }\right)}{S_{n}}\right)
$$

\subsubsection{Similarity Index (SI)}

The proposed application, check originality of the enhanced image by comparing it against the original image and subsequently producing a similarity index. This similarity index contains detailed information about the number of and the use of pixel from the primary image (original image) that produced the enhanced image. The Similarity Index is a percentage of pixels in the enhanced image that match the pixels in the primary or original image. Similarity Index (SI) ranges from 0 to 1 where 0 represents $0 \%$ and 1 represents $100 \%$. SI is equal to 1 if both images are identical (original image image1-K (i, j) and enhanced image image2- I (i, j)). According to literature, it is proposed that an enhanced image is considered to be similar to the original image when the Similarity Index is greater than $40 \%$ when measured in terms of percentages, or greater 0.4 when measured in ones. Similarity Index (SI) measure is given by the formula below, where $\mathrm{x}$ and $\mathrm{y}$ denote the mean values of images original image image $1-\mathrm{K}(\mathrm{i}, \mathrm{j})$ and enhanced image image $2-\mathrm{I}(\mathrm{i}, \mathrm{j})$ and $\mathrm{ma} 2, \mathrm{mb} 2$, and mab denotes the variance of image $1-\mathrm{K}(\mathrm{i}, \mathrm{j})$, image 2- I (i, j) and covariance of image1-K $(i, j)$ and image2I $(i, j) .[15]$

$$
S I=\frac{m_{a b} 2 x y 2 m_{a} m_{b}}{m_{a} m_{b} x^{2}+y^{2} m_{a}^{2}+m_{b}^{2}}
$$

\subsubsection{Image Quality Index (IQI)}

Image quality index (IQI) is a universal or standard measure for the differences in pixels, between two images (image $1 \&$ image2) and its value ranges from -1 to 1 . IQI is equal to 0 if both images are identical (original image image 1-K (i, j) and enhanced image image $2-I(i, j))$. It is the opposite of SI when images are identical. If IQI is less than but closer to 1 (say 0.8704), then the quality of the enhanced image is described as better since the enhanced image is interpreted by the processing algorithm as being $87.04 \%$ different in defects to the original image but only $12.96 \%((1-0.8704) * 100 \%)$ similar in defects from the 
original image. (This $12.96 \%$ is also the Similarity Index) However, if the resultant image quality index (IQI) is less than but distant from 1 (say, 0.1406), then the enhanced image is described as poor since the enhanced image is interpreted by the processing algorithm as being only $14.06 \%$ different to the original image, but $85.94 \%((1-0.1406) * 100 \%)$ similar from the original image (This $85.94 \%$ is also the Similarity Index. Here, the IQI is much more interpretable as good or poor, and identical or different, if the resultant image (enhanced image) is visually appealing by the human eye or very useable by a computer for further processing. IQI is calculated by subtracting SI from 1 as given in the formula below:

$$
I Q I=1-S I
$$

\subsubsection{Pearson Correlation Coefficient ( $r$ )}

Spearman Pearson Correlation, also represented as $\mathrm{r}$ can be used to determine the degree of association between two sets of rank-ordered in pixels of two images. This was used to calculate the difference between the ranking of pixel cases in the pixels of the original image, and the ordering in the set of pixels forming the enhanced image. This is effectively the same as minimizing the mean square error between the desired rankings and the obtained rankings. Also the $\chi^{2}$ (chisquare) statistic can be used to measure the degree of association between two images in terms of its sets of pixels, but this statistic does not contribute to minimizing the mean square error between the desired rankings and the obtained rankings. The Correlation Coefficient method is used to determine how closely the input and output images co-vary. Correlation coefficient is widely used for comparing images. It is widely used in statistical analysis, pattern recognition, and image processing. The formula for the calculation of the Pearson Correlation Coefficients ( $r$ ) is given as follows, where, $\mathrm{i} 1$ is the intensity of the ith pixel in image $1, \mathrm{j} 1$ is the intensity of the ith pixel in image $2, i$ is the mean intensity of image 1 and $\mathrm{j}$ is the mean intensity of image2. [13]

$$
\text { Correlation }\left(i j, i_{1} j_{1}\right)(r)=\frac{\sum_{i=1}^{n}\left(i_{1}-i\right)\left(j_{1}-j\right)}{\sqrt{\sum_{i=1}^{n}\left(i_{1}-i\right)^{2}} \sqrt{\sum_{i=1}^{n}\left(j_{1}-j\right)^{2}}}
$$

It is a single summary number that tells you whether a relationship exists between two image pixels, how strong that

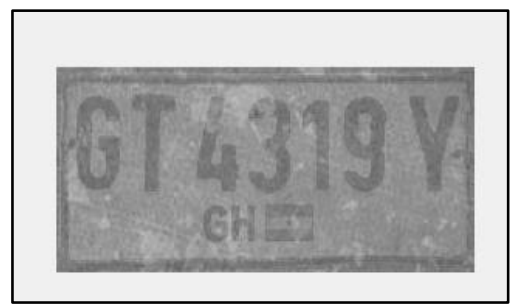

(b)

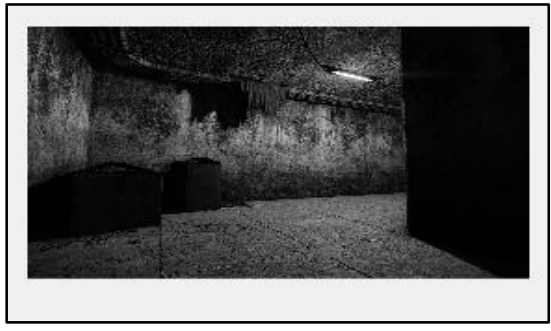

(d) relationship is and whether the relationship is positive or negative.

All correlation values are being between + or -1.00 . If " $r=$ 0.00 " that would mean that there was no relationship at all between the pixels from the original image and the pixels from the enhanced image. If the Correlation value is closer to 1 , (say $r=0.9360$ ), the pixel values of the original image is said to have a very strong relationship with the pixel values of the enhanced images. In other words, accurate predictions about how pixels from the original image are left in the enhanced images could be made. Also, if the Correlation value is slightly greater than to 0.5 , (say $r=0.6660$ ), the pixel values of the original image is said to have a strong relationship with the pixel values of the enhanced image. A weak relationship between the pixel values is when the correlation value is lower than to 0.5 (say $r=0.2660$ ).

\subsubsection{Mean Absolute Error (MAE)}

It is the Difference between original and enhanced image and is given as

$$
M A E=\frac{1}{n} \sum_{i=0}^{n}\left|f_{i}-y_{i}\right|=\frac{1}{n} \sum_{i=1}^{n}\left|e_{i}\right|
$$

As the name suggests, the mean absolute error is an average of the absolute errors $e_{i=}\left|f_{i}-y_{i}\right|$, where $f_{i}$ is the prediction and $y_{i}$ the true value. Note that alternative formulations may include relative frequencies as weight factors. The mean absolute error used the same scale as the data being measured. This is known as a scale-dependent accuracy measure and therefore cannot be used to make comparisons between series using different scales.

Or simply,

$$
M A E=|E(x)-E(y)|
$$

Where $\mathrm{E}(\mathrm{x})=$ average intensity of input image $\mathrm{E}(\mathrm{y})=$ average intensity of enhanced image

\section{ANALYSIS}

Four test images are used in this paper for the analysis which are Car Number.jpg, Checker Floor.jpg, Dark Room.jpg and Fingerprint.jpg as shown in Fig. 1. The four images are being tested on, with the three contrast enhancement techniques were compared by checking their computed PSNR, SNR, MSE, MAE, SI, IQI, RMSE and the Pearson correlation coefficient (r) values.

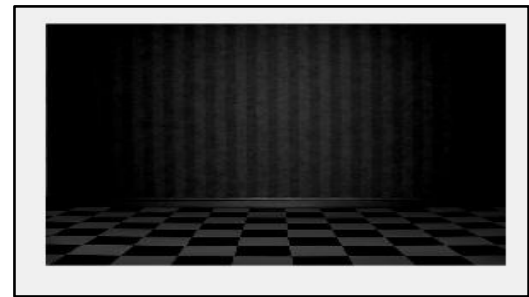

(c)

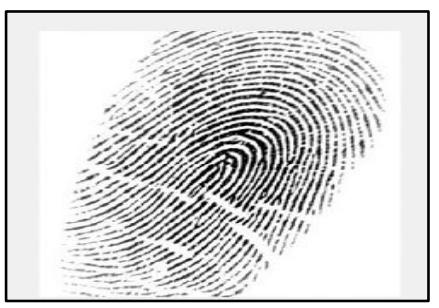

(e)

Fig. 1: Test Images. (a) Car Number.jpg. (b) Checker Floor.jpg (c) Dark Room.jpg (d) Fingerprint.jpg 


\subsection{Contrast Enhancement Techniques}

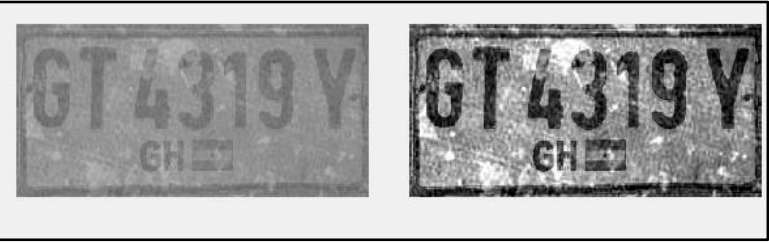

(a)

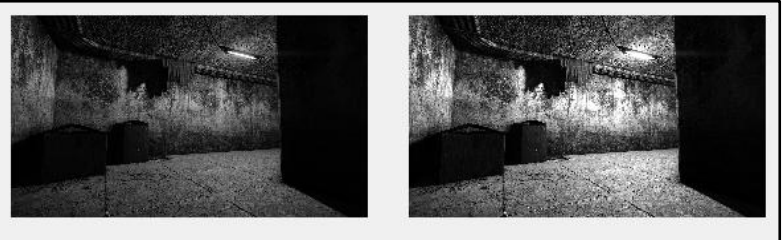

(c)
The output when the three contrast enhancement technique are applied are shown in Fig. 2 to Fig 4 with the original and enhanced images showed side by side.

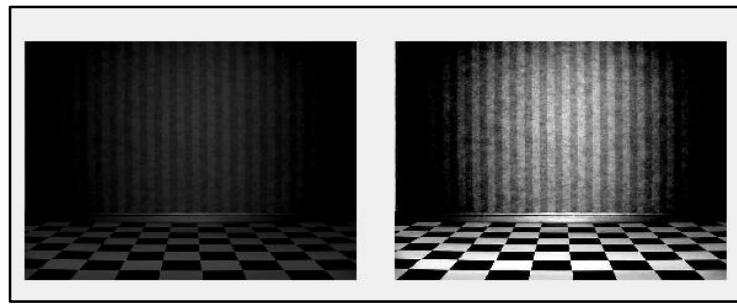

(b)

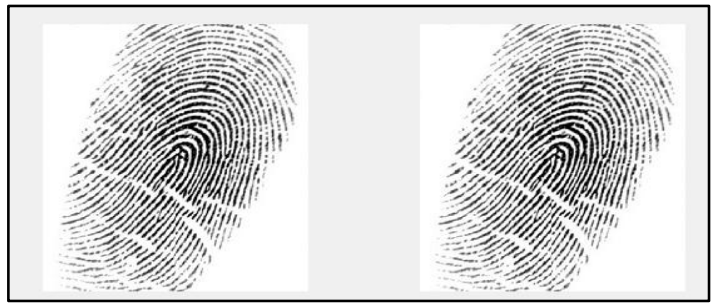

(d)

Fig. 2: Adjustment Contrast Enhancement Technique applied on Test Images. (a) Car Number.jpg. (b) Checker Floor.jpg (c) Dark Room.jpg (d) Fingerprint.jpg

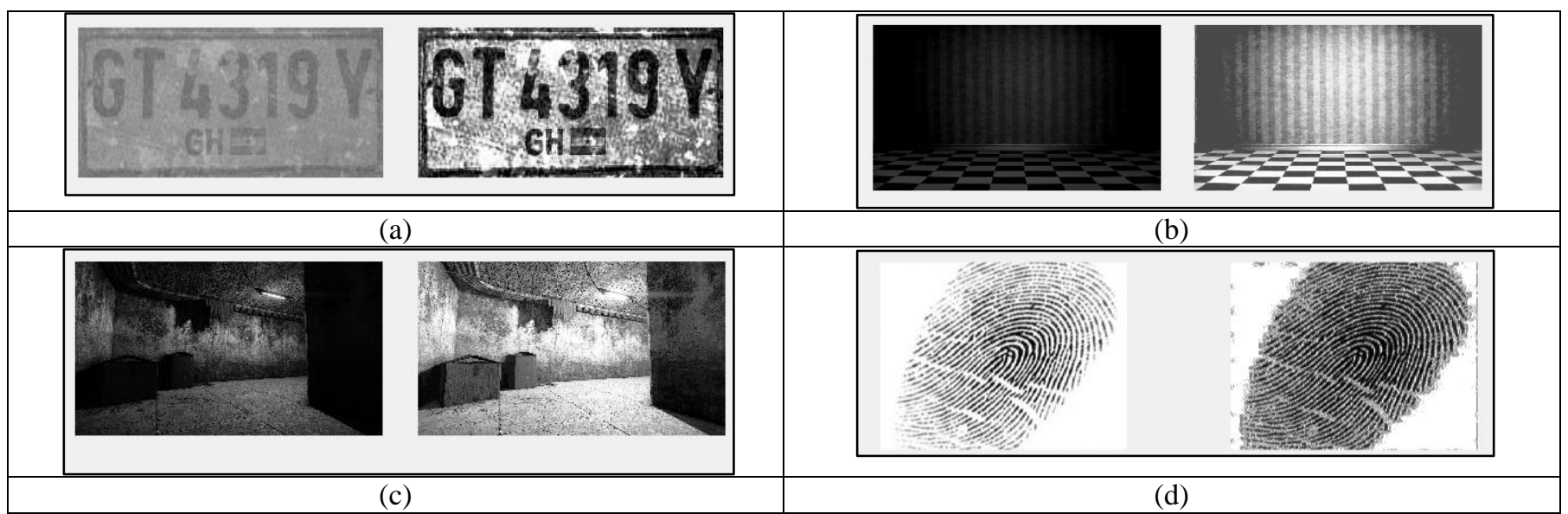

Fig. 3: Histogram Equalization Contrast Enhancement Technique applied on Test Images. (a) Car Number.jpg. (b) Checker Floor.jpg (c) Dark Room.jpg (d) Fingerprint.jpg

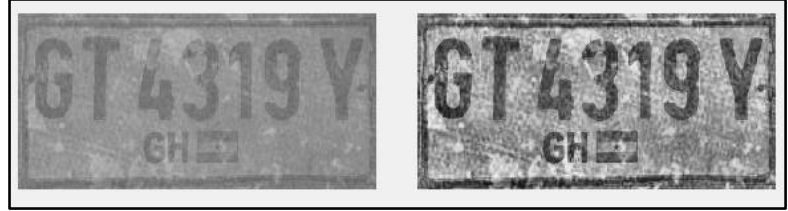

(a)

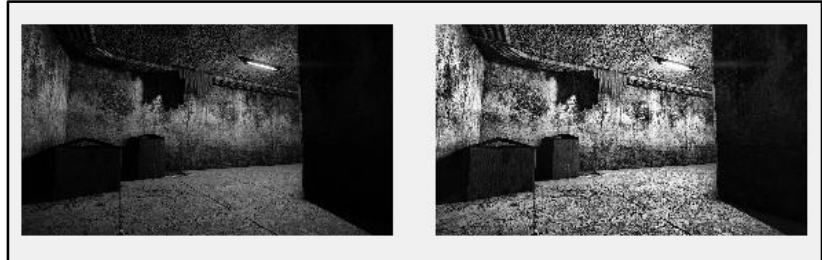

(c)

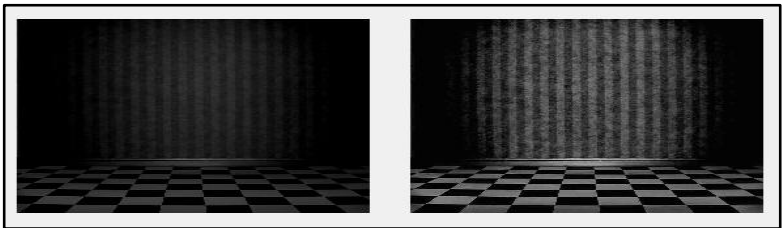

(b)

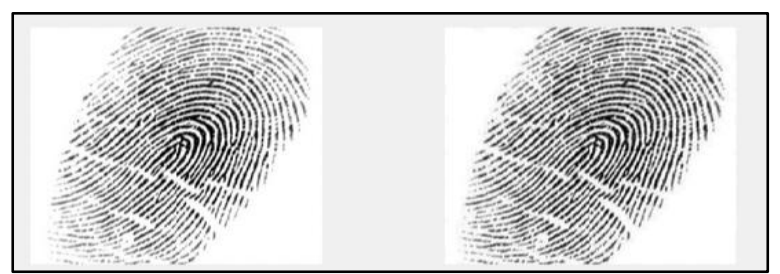

(d)

Fig..4: Contrast-Limited Histogram Equalization Technique applied on Test Images. (a) Car Number.jpg. (b) Checker Floor.jpg (c) Dark Room.jpg (d) Fingerprint.jpg 


\subsection{Measuring Performance of Enhancement Techniques}

Table 1: Enhancement Comparison between three contrast enhancement methods on test image 'Car Number.jpg'

\begin{tabular}{|l|c|c|c|}
\hline \multirow{2}{*}{ Performance Metrics } & \multicolumn{3}{|c|}{ Test Image 1 (Car Number.jpg) } \\
\cline { 2 - 4 } & AHE & HE & CLAHE \\
\hline SI & $44.2857 \%$ & $\mathbf{3 1 . 8 4 0 4 \%}$ & $53.9318 \%$ \\
\hline MSE & $2164.44 \%$ & $3712.17 \%$ & $911.303 \%$ \\
\hline IQI & $55.743 \%$ & $\mathbf{6 8 . 1 5 9 6 \%}$ & $46.0682 \%$ \\
\hline RMSE & $46.5235 \%$ & $60.9276 \%$ & $10.1878 \%$ \\
\hline PSNR & $85.1886 \%$ & $87.5315 \%$ & $81.4318 \%$ \\
\hline SNR & $14.2888 \%$ & $16.6309 \%$ & $10.5312 \%$ \\
\hline MAE & $36.2562 \%$ & $53.0597 \%$ & $25.3267 \%$ \\
\hline R & 0.800111 & 0.796472 & 0.788608 \\
\hline Visual Appearance & Good & Very Good & Good \\
\hline
\end{tabular}

When the contrast adjustment image enhancement technique was applied to the test image "Car number.jpg", the IQI and SI were $55.743 \%$ and $44.2857 \%$ respectively, which interprets that, the image was improved by a signal percentage of $55.743 \%$ when compared to the original car plate image that was imported, but was $44.285 \%$ similar to this same original image. Also, when the contrast histogram equalization technique was applied to the test image, the IQI and SI were $68.2857 \%$ and $31.8404 \%$ respectively which interprets that, the image was at a very low percentage $(31.8404 \%)$, similar to the old original car plate image, while, the technique improved the quality of the image by a signal percentage of $68.2857 \%$ when compared to the original car plate image that was imported.
When the contrast limited adjustment histogram equalization technique was applied on the test image, 'Car Number.jpg', the IQI and SI parameters, recorded $46.0682 \%$ and $53.9318 \%$ respectively. This shows that, although the image was enhanced at $46.0682 \%$, it was at a very high percentage of $53.9318 \%$, similar to the original car plate image that was imported.

From the Table 1, HE produced high IQI value of $68.1596 \%$ and a less value of SI of $31.8404 \%$. Higher value of IQI for HE means that the image was much better and much more presentable visually and signally, when histogram equalization contrast enhancement technique was applied on the image. With this, it was also realized that there were high percentage values of the enhancement parameters when HE was applied on the test image 'Car Number.jpg'.

Table 2: Enhancement Comparison between three contrast enhancement methods on test image 'Checker Floor.jpg'

\begin{tabular}{|l|c|c|c|}
\hline \multirow{2}{*}{ Performance Metrics } & \multicolumn{3}{|c|}{ Test Image 2 (Checker Floor.jpg) } \\
\cline { 2 - 4 } & AHE & HE & CLAHE \\
\hline SI & $35.8183 \%$ & $\mathbf{1 0 . 6 4 8 6 \%}$ & $30.7723 \%$ \\
\hline MSE & $5977.87 \%$ & $16486.1 \%$ & $1043.81 \%$ \\
\hline IQI & $64.1817 \%$ & $\mathbf{8 9 . 3 5 1 4 \%}$ & $69.2277 \%$ \\
\hline RMSE & $77.3167 \%$ & $128.398 \%$ & $32.308 \%$ \\
\hline PSNR & $89.6007 \%$ & $94.0064 \%$ & $13.8806 \%$ \\
\hline SNR & $21.4599 \%$ & $25.8656 \%$ & $24.7421 \%$ \\
\hline MAE & $54.1433 \%$ & $120.756 \%$ & 0.972632 \\
\hline R & 0.987261 & 0.983433 & Good \\
\hline Visual Appearance & Good & Very Good & \\
\hline
\end{tabular}

After the contrast adjustment image enhancement technique was applied to the test image 'Checker Floor', the IQI and SI were computed to be $64.1817 \%$ and $35.8183 \%$ respectively. This means that the image was only $35.8183 \%$ different and better in quality that the original image but was at a high percentage of $64.1817 \%$, similar to the original image. Also, when the contrast histogram equalization technique was applied to the test image, the IQI and SI were $89.3514 \%$ and $10.6486 \%$ respectively which interprets that, the image was at a very low percentage $(10.6486 \%)$, similar to the old original car plate image, while, the technique improved the quality of the image by a signal percentage of $89.3514 \%$ when compared to the original floor image that was imported. Lastly after the contrast limited adjustment histogram equalization technique was applied on the test image,
'Checker Floor.jpg', the IQI and SI parameters, recorded $30.7723 \%$ and $69.2277 \%$ respectively. This shows that, the image although the image was only enhanced at $30.7723 \%$, it was at a very high percentage of $69.2277 \%$, similar to the original floor image that was imported.

From Table 2, also, HE computed a very high values the eight-performance metrics and visually, the appearance of the image after contrast enhancement techniques was applied, was very good. From the table HE calculated high IQI value of $89.3514 \%$ and a less value of SI of $10.6486 \%$. Higher value of IQI and other performance metrics for HE means that the image was much better in quality, when histogram equalization contrast enhancement technique was applied on the test image 'Checker Floor.jpg. 
Table 3: Enhancement Comparison between three contrast enhancement methods on test image 'Dark Room.jpg'

\begin{tabular}{|l|c|c|c|}
\hline \multirow{2}{*}{ Performance Metrics } & \multicolumn{3}{|c|}{ Test Image 3 (Dark Room.jpg) } \\
\cline { 2 - 4 } & AHE & HE & CLAHE \\
\hline SI & $55.1963 \%$ & $\mathbf{3 1 . 1 3 6 4 \%}$ & $38.3117 \%$ \\
\hline MSE & $1996.38 \%$ & $9133.59 \%$ & $2919.27 \%$ \\
\hline IQI & $44.8037 \%$ & $\mathbf{6 8 . 8 6 3 6 \%}$ & $61.6883 \%$ \\
\hline RMSE & $44.6809 \%$ & $95.5698 \%$ & $54.0303 \%$ \\
\hline PSNR & $84.8376 \%$ & $91.4416 \%$ & $86.4879 \%$ \\
\hline SNR & $15.7729 \%$ & $22.3769 \%$ & $17.4232 \%$ \\
\hline MAE & $32.2751 \%$ & $86.8471 \%$ & $41.2645 \%$ \\
\hline R & 0.648882 & 0.554819 & 0.532274 \\
\hline Visual Appearance & Good & Very Good & Good \\
\hline
\end{tabular}

After the contrast adjustment image enhancement technique, histogram equalization image enhancement technique, contrast limited histogram equalization, were applied to the test image "Dark room", the 'Image Quality Index (IQI)' and the 'Similarity Index, (SI)' were computed to be $44.8037 \%$ and $55.1963 \%, 68.8636 \%$ and $31.1364 \%$ and $38.3117 \%$ and $61.6883 \%$ for respectively. This means that the image was better increased when the histogram equalization image enhancement technique was applied on the test image 'Dark Room.jpg' because the recorded value for the IQI was $68.8636 \%$ whilst the computed percentage of the SI was $31.1364 \%$. This shows that the enhanced image was about $31 \%$ similar to the original image whilst the same enhanced image was about $69 \%$ better than the originally imported image.

Again, from Table 3, also, HE computed a very high values for all the eight-performance metrics and visually, the appearance of the image after histogram equalization contrast enhancement techniques was applied, was very good. From the table HE calculated high IQI value of $68.8636 \%$ and a less value of SI of $31.1364 \%$. Higher value of IQI and other performance metrics for HE means that the image was much better in quality, when histogram equalization contrast enhancement technique was applied on the test image 'Dark Room.jpg'.

Table 4: Enhancement Comparison between three contrast enhancement methods on test image 'Fingerprint.jpg'

\begin{tabular}{|l|c|c|c|}
\hline \multirow{2}{*}{ Performance Metrics } & \multicolumn{3}{|c|}{ Test Image 3 (Fingerprint.jpg) } \\
\cline { 2 - 4 } & AHE & HE & CLAHE \\
\hline SI & 99.1562 & $\mathbf{3 1 . 8 8 1 1}$ & 93.274 \\
\hline MSE & 37.7343 & 9010.6 & 397.077 \\
\hline IQI & 0.84378 & $\mathbf{6 8 . 1 1 8 9}$ & 6.72596 \\
\hline RMSE & 6.14283 & 94.9242 & 19.9268 \\
\hline PSNR & 22.3974 & 91.3827 & 77.824 \\
\hline SNR & 8.27831 & 20.5019 & 6.94307 \\
\hline MAE & 2.66582 & 56.5602 & 10.0588 \\
\hline R & 0.98748 & 0.867778 & 0.976694 \\
\hline Visual Appearance & Good & Very Good & Good \\
\hline
\end{tabular}

The contrast histogram equalization technique gave a SI and IQI of $31.8811 \%$ and $68.1189 \%$ respectively which interprets that, the image was at lower percentage $(31.8811 \%)$, similar, than it is different, to the old original fingerprint image.

Also when the contrast limited adjustment histogram equalization technique was applied on the test image, 'Fingerprint.jpg', the IQI and SI parameters, recorded $6.72596 \%$ and $93.274 \%$ respectively. This shows that, the image was only enhanced at about $7 \%$, but was similar to the originally imported fingerprint image at a very high percentage of about $93 \%$. In other words, the contrast limited adjustment histogram equalization technique did not increase the quality of the image at all.

From the Table 4, HE produced high IQI value of $68.1189 \%$ and a less value of SI of $31.8811 \%$. Higher value of IQI for HE means that the image was much better and much more presentable visually and signally, when histogram equalization contrast enhancement technique was applied on the image. With this, it was also realized that there were high percentage values of the enhancement parameters when HE was applied on the test image 'Fingerprint.jpg'.

\section{CONCLUSION}

The performances of the image enhancement techniques were evaluated in terms of eight enhancement metrics and the visual quality, to quantify the differences between the original image and the enhanced image. The experiment was carried out to study the performance of three image enhancement schemes at different levels of image defections. These three image contrast enhancement techniques were the image contrast adjustment (AHE), histogram equalization (HE), and contrast limited adjusted histogram equalization (CLAHE) techniques The study analyses the output of these three image contrast enhancement techniques on images with eight enhancement parameters which were SI, MSE, IQI, RMSE, PSNR, SNR, MAE and $r$, but was very attentive to the percentage values of IQI and SI. In addition, the research also visually compared the enhanced images after each of the image enhancement technique was applied on the test images. In this paper, four test images were used to basically test the system's robustness. The output generated was an enhanced image improved in contrast and visualization. This output image is then compared with the original test image. The results showed that histogram equalization (HE) technique is the best image contrast enhancement technique when 
compared to image adjustment (AHE) and contrast limited adjustment (CLAHE) image contrast enhancement techniques. The results then showed that histogram equalization (HE) technique is a very good image enhancement method that can be used to appropriately reduce image defects. In other words, it can be used to enhanced images and improved their contrast and visualization. Thus, the study proved the histogram equalization as the best image processing approach for enhancing contrast in visually poor images. Since the paper concluded that, histogram equalization technique is one of the best contrast enhancement techniques in image processing, the recommended technique is therefore characterized as one technique that improves the interpretability or perception of information in images for human viewers and for further computer analysis. Also, it can be deduced that histogram equalization image enhancement technique can be used to efficiently manage the vagueness and ambiguity in an image. It can be therefore recommended that the recommended contrast enhancement technique can be embedded in any system that processes on images and output then to humans for making decisions, such as the ultra-scan sound machines.

\section{REFERENCES}

[1] Oakley, J. P., and Satherley, B. L. 1998. Improving image quality in poor visibility conditions using a physical model for contrast degradation. IEEE Transactions on Image Processing 7 , 167-179

[2] Rahman, Z., Jobson, D. J., , and Woodell, G. A. 1996. Multi-scale retinex for color image enhancement. IEEE International Conference on Image Processing.

[3] Boccignone, G., and Picariello, A. 1997. Multiscale contrast enhancement of medical images. Proceedings of ICASSP.

[4] Kim Y. T. (1997), "Contrast Enhancement Using Histogram Equalization," IEEE Transactions on Consumer Electronics, Vol 43,No.1, pp.1-8.

[5] Madhu C. D. (2014), "Preserving Contrast in Histogram Equalization Based on Adaptive Enhancement Techniques," Computer Journal of Digital Signal Processing, Vol. 14, No. 5, pp. 413-428, 2004.
[6] Hojat Y. Ali Z., Amirhossein R., (2008) “A Novel Approach for Contrast Enhancement Based on Histogram Equalization". International Conference on Computer and Communication Engineering, ICCCE, pp. $256-260$.

[7] Wang-Zhiming, TAO-Jianhua (2007), "A Fast Implementation of Adaptive Histogram Equalization" 2006 8th International Conference on Signal Processing .

[8] Rajesh G., Bhawna M., Sheetal G. (2011) "Histogram Equalization Techniques for Image Enhancement," IJECT Vol. 2, Issue 1.

[9] Confucius (2013), "Pictures, its definition and Importance in Image processing,", World academy of Science, Engineering and Technology.

[10] Jeong C. B., Kim . G., Kim T. S., Kim S. K. (2011), "Comparison of image enhancement methods for the effective diagnosis in successive whole-body bone scans", Vol.24, No.3, pp. 424-36.

[11] Cheng H. D. Shi X. J. (2004). "A simple and effective histogram equalization approach to image enhancement," Digital Signal Processing Vol.14,pp. 158-170,.

[12] Saritha K R (2016). “A Study on Image Enhancement Techniques and Performance Measuring Metrics" International Journal of Innovative Research in Computer and Communication Engineering, Vol. 4, Issue 4

[13] Abhishek T., Pramil S. (2004) "Analysis of Color Contrast Enhancement Techniques", International Journal of Emerging Technology and Advanced Engineering, Volume 4, Issue 4

[14] Gayathri S., Mohanapriya N., and Kalaavathi B., (2003) "Survey on Contrast Enhancement Techniques", International Journal of Advanced Research in Computer and Communication Engineering, Vol. 2, Issue 1.

[15] Nandhini V., Pratheepa R., N. Anjana and V. Elamaran, (2015) "A Novel Approach for Contrast Enhancement using Image Classification and Subdivision based Histogram Equalization", Indian Journal of Science and Technology, Vol. 8, Issue 29 